\title{
Proyecto de Plaza-Embarcadero en Caleta Tortel, Región de Aysén, Patagonia de Chile.
}

Paulina Andrea Medel Santibáñez

\section{Resumen:}

El proyecto de Título de Arquitectura, 2007, que se presenta tiene como objetivo central dar respuesta a necesidades reales, preservando y cuidando del medio local, tanto natural como sociocultural, dentro de la dinámica actual de cambios y apertura. Se reconoce los atributos y potencialidades propios de Caleta Tortel para preservar su identidad cultural y patrimonial a través de la consideración de la relación con su entorno, específicamente, reconociendo el vínculo entre la localidad y el mar como medio elemental que le da origen y permite su desarrollo. La "PlazaEmbarcadero" en Caleta Tortel es un conjunto de carácter público, de escala comunal y con un programa comunitario integrador y abierto para la interacción diaria y directa de los habitantes y visitantes.

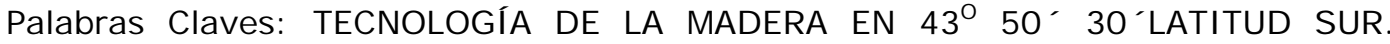
CALETA TORTEL. TIERRA DEL FUEGO, PATAGONIA DE CHILE. PROYECTO PLAZAEMBARCADERO. ARQUITECTO PAULINA ANDREA MEDEL SANTIBÁÑEZ. CIPRÉS DE LAS GUAITECAS.

\begin{abstract}
:
This work leading to the professional degree in architecture is an answer to certain needs of Caleta Tortel in the Chilean Patagonia, adapting itself to the local environment -social and natural- and to the changes of globalization. The core idea of this project is to preserve the spatial and economic link between the town and the sea. The proposed wharf is a meeting point for the neighbours and tourists.
\end{abstract}

Key word: WOOD TECHNOLOGY. CALETA TORTEL (430 50' 30’ S.). TIERRA DEL FUEGO, CHILEAN PATAGONIA.

\section{Introducción}

Para egresar de la carrera de Arquitectura, durante el segundo semestre del año 2005, la autora realizó su práctica profesional en la Secretaría Comunal de Planificación, SECPLAN, de la Municipalidad de Tortel, comuna situada entre los Campos de Hielos Norte y Sur, en la XI Región de Aysén, Chile (entre los $73^{\circ}$ y $74^{\circ}$ longitud, y entre los $47^{\circ}$ y $48^{\circ}$ latitud sur, entre Campos de Hielo Norte y Campos de Hielo Sur). Viviendo durante tres meses en el lugar, uno de los objetivos fue percibir la especificidad de la localidad, una de las bases fundamentales para el desarrollo de cualquier proyecto de arquitectura y urbanismo. Posteriormente, realizó su proyecto de título, fundamentándolo en dicha experiencia, reconociendo y aplicando luego sus atributos y potencialidades en la proposición arquitectónica. 


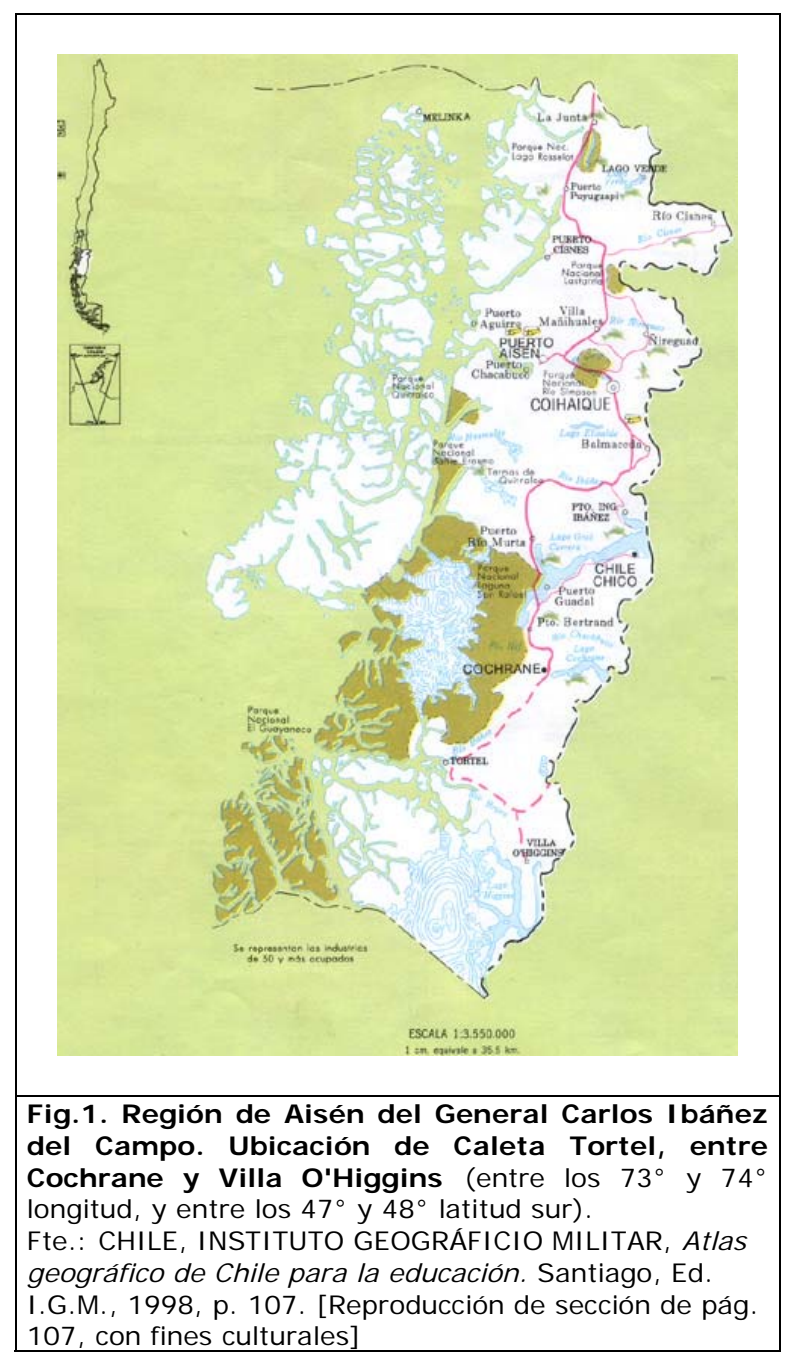

Es así que el proyecto radica en la motivación personal de la autora de poder hacer trascender y perdurar en el tiempo las características socio-culturales y ambientales locales, en el marco de una dinámica nacional (y mundial) que se enfoca principalmente al desarrollo económico, y que, en general, tiende a homogeneizar y a explotar dichas características, sin la sostenibilidad necesaria, ni una planificación de futuro.

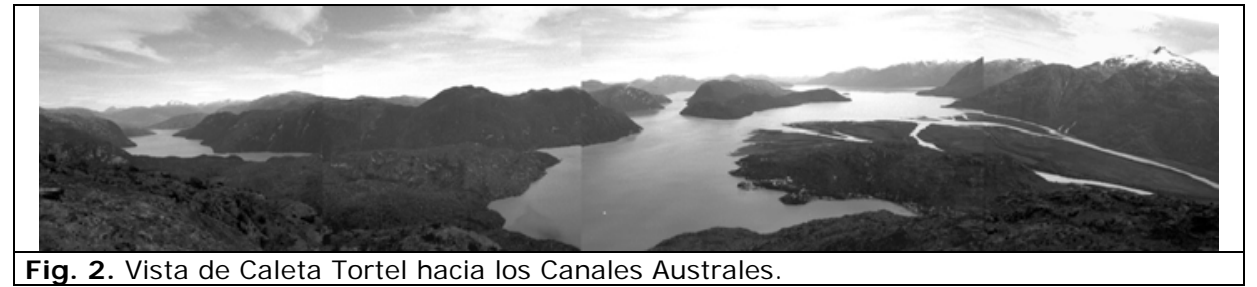

\section{Aysén, región extrema y aislada de Chile, con potencialidades para el desarrollo.}

El aislamiento del territorio de Aysén, producto de ubicación y de sus características geográficas, ha sido determinante en su historia, marcando los distintos procesos que fueron formando lo que hoy se conoce como "Región de Aysén" y sus 
localidades. El poblamiento ${ }^{1}$ de este extenso territorio chileno ha obedecido, en gran medida, a procesos autónomos de ocupación y organización, llevados a cabo por colonos espontáneos arribados desde otras regiones chilenas.

El diario vivir en la Región de Aysén, y en particular en sus localidades más aisladas, presenta una gran riqueza social, humana y del medio natural, con condiciones de vida propias de un sistema que se ha desarrollado en el aislamiento, en la soledad, con el clima adverso. Lugares comunes son, por ejemplo, aquellos en donde la cooperación y solidaridad se hace indispensable para la subsistencia, en donde la vinculación de los habitantes se ha dado en cierta intensidad con Argentina -trayendo con ello un ascendente socio-cultural muy marcado-, y donde la relación con el resto de Chile no ha sido fácil.

Nuevas condiciones comienzan a desarrollarse en la Región en las últimas tres décadas producto de la regionalización de $1974^{2}$. Este nuevo marco ha llevado al aumento de los servicios, a un incremento en la conectividad dentro del territorio chileno producto de la construcción de la carretera austral, y a una explotación de los recursos y sus características culturales. Esta situación hace de la región un medio muy atractivo tanto para la inversión turística como productiva. Se puede afirmar, así, que la región se encuentra todavía en proceso de adaptación a los cambios.

\section{Tortel: localidad atractiva de la región}

Aysén posee recursos naturales de belleza y pureza excepcionales difíciles de encontrar en otros lugares del mundo. Esto la convierte en una de las regiones más atractivas y con mayor potencial turístico a nivel nacional e internacional. Tortel es uno de estos lugares que, además de ser atractivo naturalmente, posee un asentamiento urbano particular que, en conjunto, ha significado la promulgación oficial de ella como "Zona Típica y Pintoresca" el 25 de mayo de año 2001 (Consejo de Monumentos Nacionales de Chile).

Se trata en este tipo de promulgación, de mantener y proteger el carácter ambiental y propio de ciertas poblaciones o lugares, o determinadas zonas, en donde su conservación interesa a la historia nacional.

Para Tortel, esta caracterización también significa un reconocimiento a la exteriorización cultural maderera del lugar, cuyo principal exponente está constituido por las pasarelas de ciprés de los mallines o "turberas", y la inserción de lo construido en armonía con la naturaleza. El énfasis está entonces en la conservación y preservación conjunta del patrimonio natural, cultural y arquitectónico. Estos valores y exteriorizaciones constituyen algo muy escaso y en vías a desaparecer en el mundo actual.

Por otra parte, debe destacarse la importancia de la actividad turística para la comuna ya que, debido al entorno paisajístico y cultural que posee, ha logrado posesionarse como actividad de interés a la economía del lugar económica en aumento paulatino. El sostenido crecimiento en la llegada de turistas, se va dando principalmente con la conexión con la Ruta Austral en el año 2001. Es así como la comuna de Tortel, con vocación inicial de centro de servicios, ha ido generando una mayor atracción turística, y no sólo por su belleza paisajística y atributos

\footnotetext{
1 A fines del Siglo XIX comenzó la ocupación de la Región, en los valles de los ríos Aysén, Simpson, Cisne y la cuenca del Lago General Carrera. En el Siglo XX comenzaron actividades económicas de mayor volumen, lideradas por compañías ganaderas, que permitieron que la Región fuese definitivamente colonizada. Hacia 1930 comenzó la retirada de las compañías ganaderas y también marca el fin de la colonización espontánea e individual. De allí en adelante, la acción del Estado para radicar población e invertir en obras públicas será dominante.

Proceso de división político administrativa del territorio en 13 regiones y 51 provincias, en el año 1974. Esta estructura se consolidó en la Constitución Política de 1980 y fue parte del modelo de Descentralización de Chile junto con la Municipalización.
} 
arquitectónicos sino, también, por su ubicación estratégica como punto medio entre los Campos de Hielo Norte y Sur, y sus respectivos ventisqueros, Steffens y Montt. Además, se encuentra en medio de reservas y parques nacionales. Es así que Caleta Tortel adquirió la denominación de patrimonio nacional ("Zona Típica y Pintoresca") y la llamada "Isla de los Muertos", la denominación de "Monumento Histórico".

\subsection{Población}

El origen del asentamiento combina los conocimientos del hombre ganadero de la pampa en la Patagonia, del chilote eminentemente marinero y constructor, y del hombre indígena kaweskar de los canales y tehuelches de la pampa. La herencia cultural se presenta en un nuevo contexto en donde surgen otros conocimientos que de forma natural se desarrollan según las exigencias del lugar. De esta forma se va creando una nueva cultura la que se traduce en una interacción hombre, costumbres, naturaleza, y se representa en una forma particular de asentarse y relacionarse en el territorio.

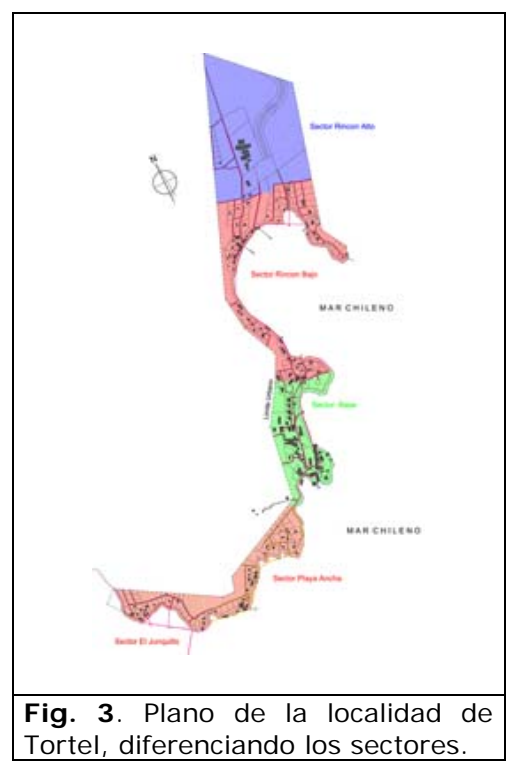

La Comuna de Tortel posee una población de 507 habitantes según el Censo del año 2002. Esta se encuentra principalmente asentada en su única localidad, Caleta Tortel (sobre el $70 \%$ de la población), encontrándose el resto de los habitantes ubicados en zonas eminentemente rurales, dispersas en el territorio comunal, muchos con un altísimo nivel de aislamiento.

En la localidad propiamente tal, se presentan tres tipos de permanencia en el centro poblado que permiten definir la potencial demanda de ocupación de suelo y la orientación del desarrollo de actividades que logren satisfacer las necesidades de la población:

- la población permanente que se caracteriza por ser colonos o descendientes directos de ellos, que se encuentra habitando Caleta Tortel de manera constante por razones de tipo familiar, laboral o por sentido de propiedad;

-la población migratoria de carácter colonizadora, que habita por temporadas el centro poblado por razones de tipo laboral, familiar, académicas, o por tener propiedades en dos o más localidades, y 
-la población flotante, que está conformada principalmente por turistas que visitan la localidad estacionalmente.

\subsection{Contexto patrimonial}

La declaración de Caleta Tortel como "Zona Típica y Pintoresca" implica que este lugar queda bajo tuición del Consejo de Monumentos Nacionales y las obras que en él se realicen serán reguladas por el Instructivo de Intervención, para resguardar el estilo arquitectónico general y otros aspectos de dicha zona.

Este Instructivo ${ }^{3}$ "define las condiciones de intervención, conservación o construcción de edificaciones para el sector enmarcado como Zona Típica "Caleta Tortel", tanto para las obras existentes a partir de la fecha de la declaratoria final, como para las nuevas que allí se ejecuten, en terrenos públicos y/o privados". (Elaborado por el Ministerio de Obras Públicas, a través de la Dirección de Arquitectura, de su Secretaría Regional Ministerial, SEREMI ).

Los criterios principales que contiene el instructivo para los inmuebles existentes que poseen valor patrimonial dicen relación con la conservación de su estado constructivo, y con la integración armónica de posibles ampliaciones con la obra existente y el paisaje natural. Para las construcciones nuevas se deberá procurar mantener las proporciones, ritmos, alturas y materialidad presentes en la Zona Típica.

El Instructivo de Zona Típica (IZT), es el único instrumento referencial al momento de diseñar, ya que la comuna no tiene Plan Regulador (PRC). Cabe considerar que dicho instructivo se encuentra en proceso de revisión a la espera de ser aprobado por el Consejo de Monumentos en Santiago, pero, para efectos del Proyecto, se tomará como herramienta válida. También hay que considerar la ejecución de obras, programas y actividades a desarrollarse en estos lugares protegidos queda sujeta a evaluación de impacto ambiental (COREMA Y CONAMA).

\subsection{Contexto social}

La condición socioeconómica de la comuna nos muestra (según Fichas CAS) que sobre un $50 \%$ de la población comunal se encuentra en una situación de pobreza, y alrededor de un $27 \%$ en condición de extrema pobreza.

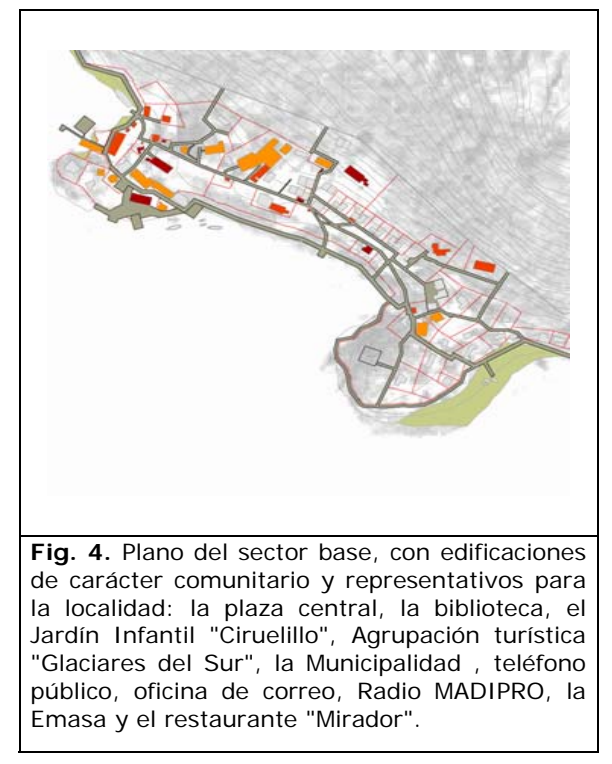

${ }^{3}$ Instructivo de Intervención: "Bases técnicas de diseño para el instructivo de intervención de la Zona típica Caleta Tortel" 
En relación con el equipamiento y servicios básicos en Caleta Tortel, es posible señalar que cuenta con una red de energía eléctrica, correo, red telefónica pública, acceso a radio, televisión e Internet, una posta rural, una escuela: 90 alumnos, un jardín infantil: 15 alumnos, comercio menor y equipamiento comunitario básico, como una plaza, dos capillas, un aeródromo, 15 embarcaderos domiciliarios y un muelle de embarcaciones mayores, 9 hospedajes, 4 restaurantes y 1 panadería. Como no se cuenta con una planta de tratamiento de aguas servidas, los desechos se evacuan directamente al mar, en las costas frente a la localidad, lo que ocasiona problemas de sanidad y malos olores (actualmente se gestiona una planta de tratamiento de aguas para suplir las necesidades de un sector de Tortel).

La actual gestión pública reconoce a las personas como el activo más valioso que posee una comunidad, considerando la capacitación y el mejoramiento organizacional como herramientas primordiales para un desarrollo humano y productivo.

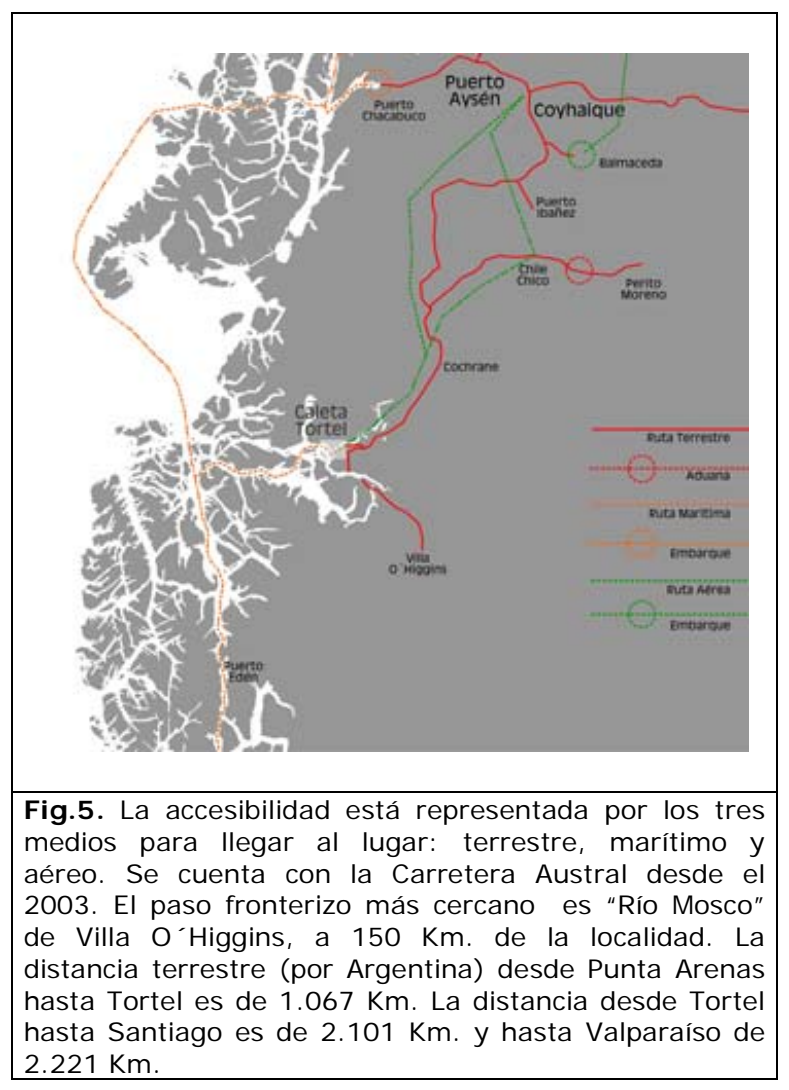

\subsection{Contexto económico}

Tortel, históricamente, tuvo su actividad centrada en la extracción maderera del ciprés de las Guaitecas. Hoy esta actividad ha disminuido por la sobreexplotación a la cual fue sometido, y se ha ido complementando y sustituyendo a veces, con actividades ganaderas, extractivas marinas en forma incipiente (pescados como el salmón, la merluza y el róbalo, y mariscos en general) y con la actividad turística con perspectivas de desarrollo. La economía de la comuna se caracteriza por la fuerte presencia gubernamental. La participación del sector comercio es de un $53 \%$, mientras que el resto de los sectores participa con un $10 \%$.

Es así que la comuna cuenta con una gran cantidad de recursos naturales, los que representan una oportunidad concreta de inserción en el ámbito económico local, 
regional, e incluso nacional, y que permiten cubrir las necesidades básicas de sus pobladores. Dentro de este marco general del proceso económico-productivo local se identifican tres ejes esenciales de desarrollo económico y productivo: sector pesquero, sector turístico, y sector silvoagropecuario.

\subsection{Contexto natural}

El territorio en el cual se encuentra la localidad, está constituida por diversos ecosistemas muy distintos pero complementarios, donde coexisten para generar una piel sobre el suelo. Algunos de estos ecosistemas que se superponen en este lugar son: sistema de glaciares (cerro Las Heras), ecosistema de aguas dulces fluviales, de inundación de juncales y plantas palustres, limnológico de laguna; ecosistema de montaña ${ }^{4}$.

El suelo donde se posa esta piel vegetal, prácticamente no existe, debido a que se encuentra en una de sus primeras etapas de evolución, luego de que se retiraran los hielos. La fragilidad de este territorio y de este terreno en particular, radica en que está en un momento de trasformación con el fin de consolidar lo que será el suelo a futuro.

Primero se posan en las rocas los líquenes, luego vienen los musgos y después las hierbas, para luego dar paso a los arbustos. Cada uno de ellos por medio de sus raíces, van destruyendo la roca para el desarrollo de un bosque y de especies más evolucionadas.

Al saber cómo es el suelo, su composición, origen y evolución, podemos entender que lo construido en la localidad ha sido una respuesta natural al medio en que se asienta, posándose sobre él sin querer alterar todo el proceso natural de la piel vegetal, conformándose como una unidad paisajística, con características ambientales propias que definen una identidad, referencia histórica y urbana.

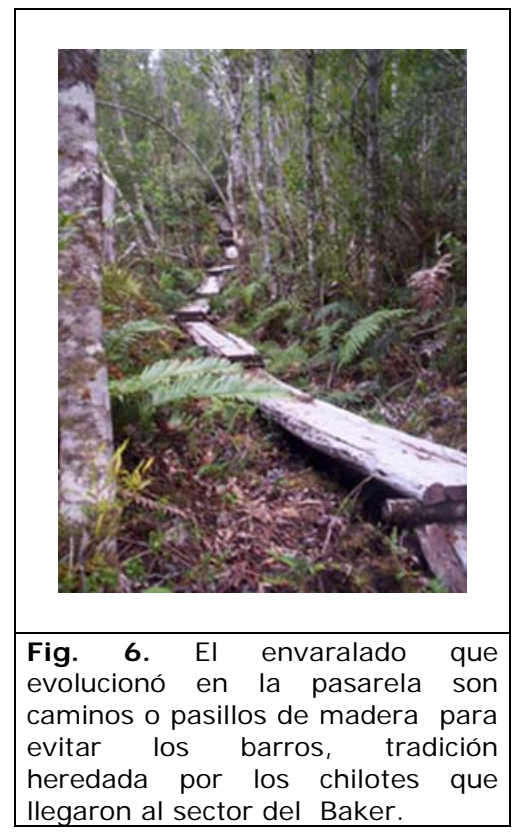

Es necesario entender el suelo como una de las condicionantes geográficas y climáticas que caracteriza el territorio: gran pluviosidad, cerca de 4000 milímetros anuales y una topografía abrupta: suelo rocoso con participación de musgos, vegetación naciente, exceso y erosión hídrica; un suelo no pisable. Sobre estas

\footnotetext{
${ }^{4}$ Renato Gres. Características de los suelos del litoral de aysén. Sociedad chilena de la ciencia y el suelo.
} 
cualidades se definió el proyecto considerando su fragilidad, el posarse y respetar su evolución.

\section{El proyecto de Plaza-Embarcadero en Caleta Tortel}

Es importante considerar la existencia de estos lugares particulares, como Tortel, con culturas específicas diferentes a la urbana, un desarrollo con medios escasos, a veces únicos, en donde la mano del hombre civilizado, la contaminación y la urbanización no han modificado el entorno. Estos lugares son, entonces, territorios con potenciales propios para el desarrollo. ¿Pero de qué manera?

Desde el punto de vista de la autora, la arquitectura debiera ser una herramienta que reconoce estas potencialidades propias del lugar y del medio natural en el cual se inserta, para así, generar oportunidades de desarrollo sostenible, autónomo, propio, que pueda mantenerse por si mismo en el tiempo.

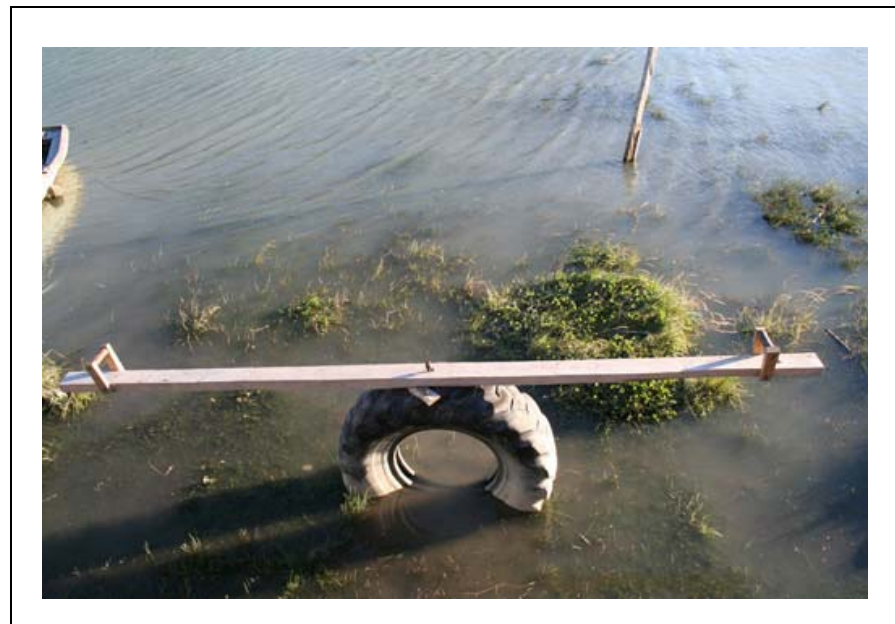

Fig. 7. Los juegos de niños están en el suelo con variación de marea, condicionando su uso diario.

La estrategia se basa entonces en una arquitectura dialogante con el territorio en el cual se inserta, reconociendo las potencialidades locales.

\subsection{Relación con el entorno social}

\section{La arquitectura dialoga con los elementos históricos}

Valor en el significado socio espacial.

-CENTRO DE LA LOCALIDAD: el proyecto reposiciona el lugar natural de confluencia de flujos y actividades, creando infraestructura necesaria representativa y potenciadora de la cultura local.

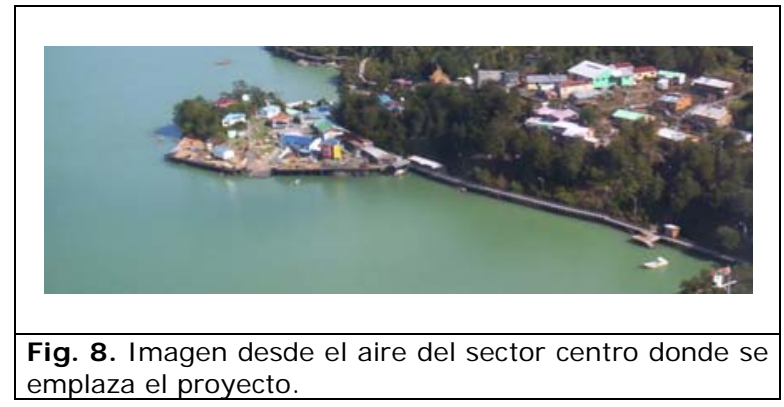


-EJE CÍVICO / EJE COSTANERA: el proyecto reconoce la estructura local que es la pasarela, generando la continuidad de ella, uniendo el eje cívico (equipamiento local) y el eje costanera (paseo Tortel)

-SIGNIFICADO HISTÓRICO DEL MAR: el proyecto reconoce la importancia del mar, por lo que significa y significó; el proyecto se emplaza en el borde.

\section{La arquitectura dialoga con los actores del proyecto}

El espacio público.

-PLAZA Y PASARELA: el proyecto reconoce la pasarela como espacio público, donde acoge los actos.

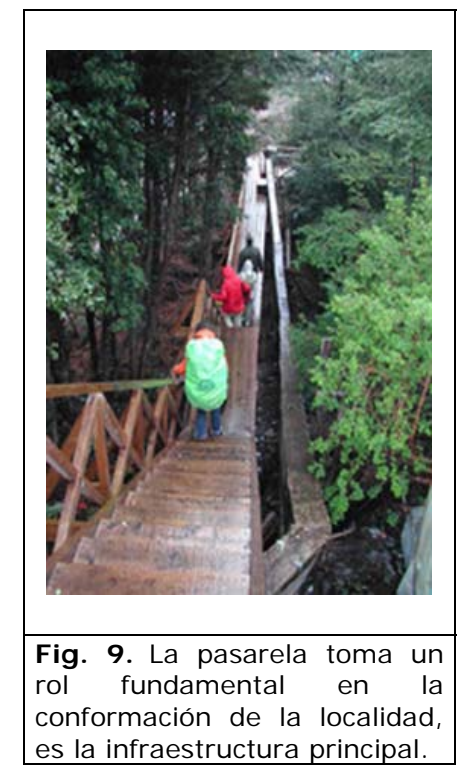

Usuarios. Reconocer los actos esenciales. La cotidianidad de la vida.

-EL HABITANTE LOCAL: el proyecto crea espacios donde se realizan las actividades cotidianas del lugar, donde se reconozcan sus habitantes, su cultura, su historia, costumbres y tradiciones.

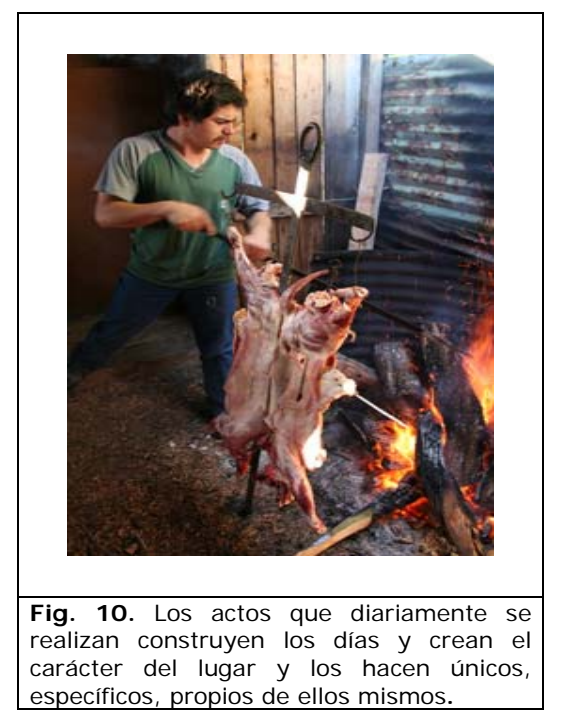


-LOS USUARIOS: el proyecto integra no sólo al habitante local, sino también al turista, a las personas del área rural de la comuna y a los estudiantes.

\subsection{Relación entorno natural}

\section{La arquitectura dialoga con los elementos del entorno.}

El paisaje, estructura natural.

- EL SUELO condición geográfica que caracteriza el territorio. Territorios suelo de ecosistemas: el proyecto se posa sobre el terreno sin alterar el proceso natural de la vegetación. También considera en los suelos erosionados un tratamiento de paisaje para regenerar la capa vegetal.

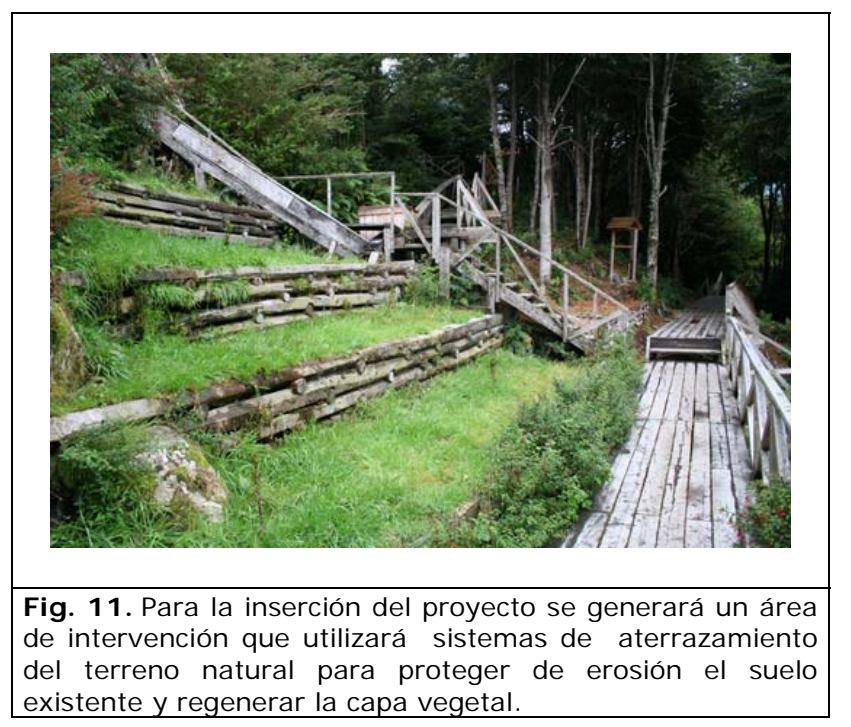

- NATURALEZA, masa impenetrable: el proyecto se emplaza en el borde.

- MAR COMO COMUNICADOR: el proyecto reconoce el rol de Tortel como punto de enlace de las rutas marítimas. En Tortel se ha creado circuitos turísticos y circuitos de traslados por conexión, además de la función de carga, descarga y almacenamiento cada vez más eventual. Estas actividades que ligan a la localidad con el mar, han adquirido dimensiones graficadas a continuación.

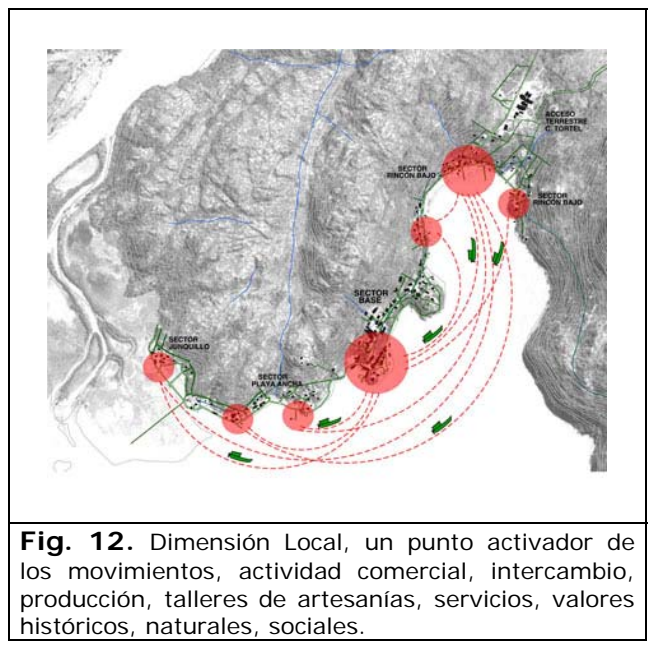




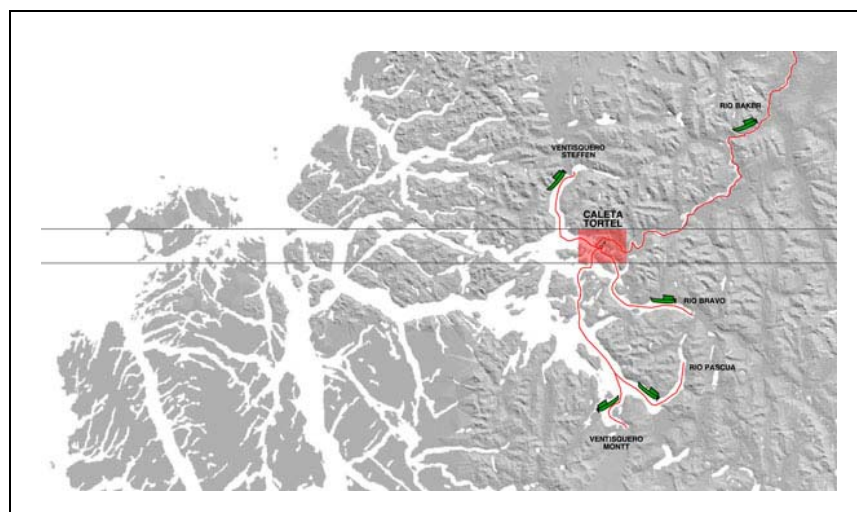

Fig. 13. Dimensión Zonal, un punto de conexión para zonas de la comuna. Desde el campo van a la localidad a abastecerse. Campos en las zonas de: Río Baker, Río Pascua, Río Bravo, Ventisquero Montt, Ventisquero Steffen.

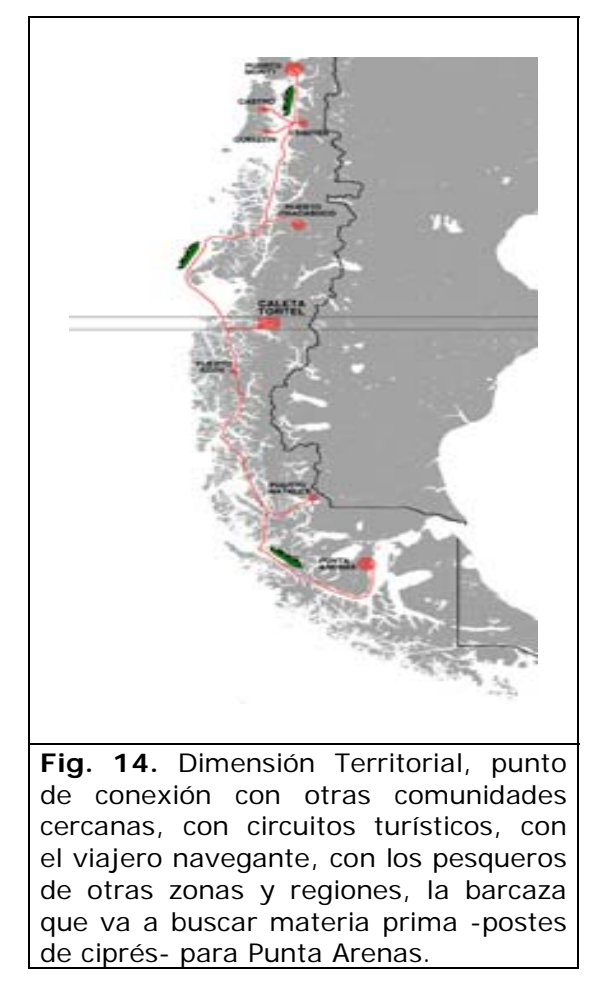

-EMBARCADERO, ARTICULADOR DE FLUJOS: el proyecto reconoce la relación de las personas con el mar en su rol de cotidianeidad. Un espacio como lugar reconocido para el embarque de pasajeros, transporte de mercaderías, viajes de turismo, etc. La activación social se traduce así en la posibilidad de crear un vínculo físico que permita este intercambio cultural, comercial y productivo. 


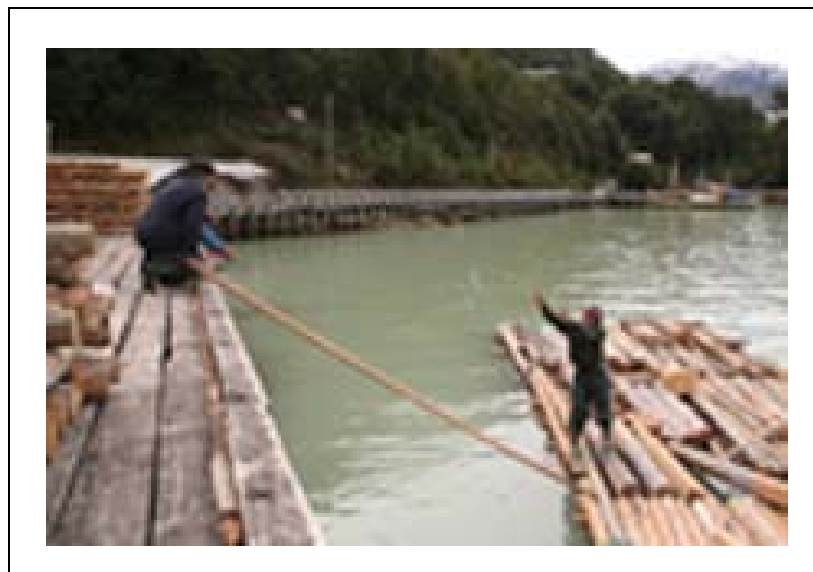

Fig. 15. Acopio en el muelle de las varas de ciprés traídas desde los campos por la balsa.
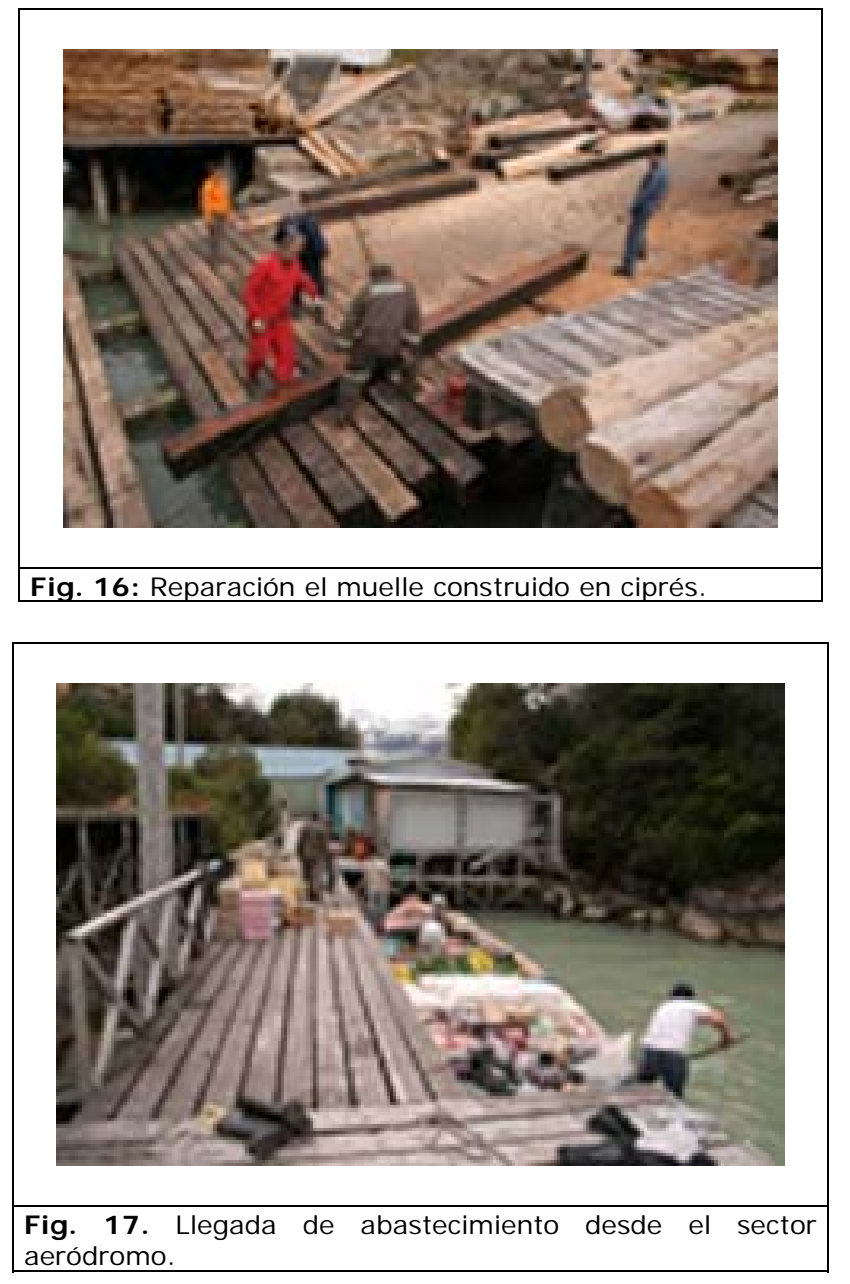

-LA PASARELA, trazo y recorrido: el proyecto reconoce la pasarela como el elemento construido vinculador de dos situaciones, el espacio marítimo como medio que sustenta y el espacio construido donde habita el poblado, el proyecto entonces pasa a ser una pasarela. 


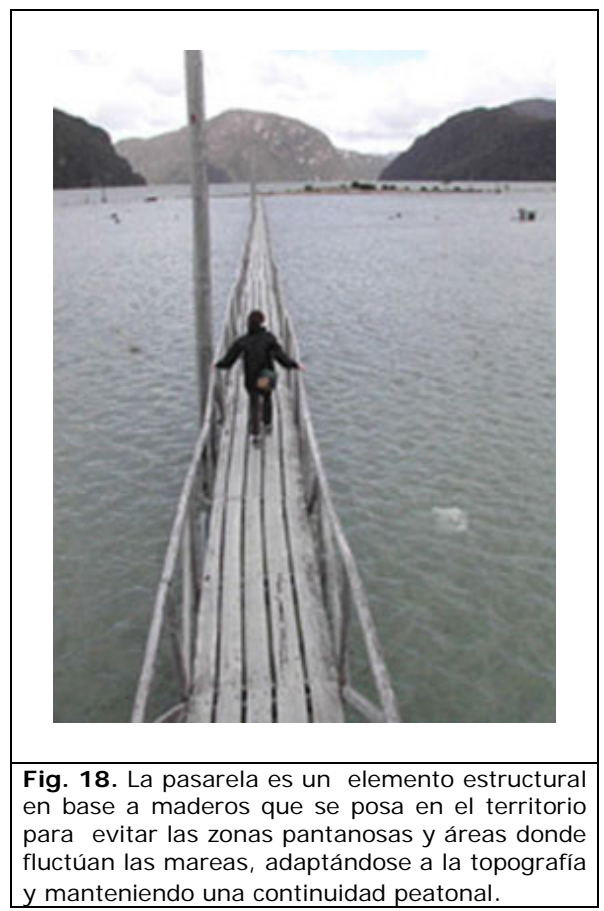

Clima.

-LLUVIA CONSTANTE, 4000 milímetros anuales, y bajas temperaturas 6/14ㅇ c promedio: el proyecto crea espacios resguardados de la lluvia y el frío, mediante construcciones con dimensiones acotadas para una rápida calefacción y espacios techados.

Materialidad.

- LA MADERA, evento y material: el proyecto aprovecha los recursos existentes del lugar y reconoce las técnicas constructivas locales. La canoga se propone como un sistema de techumbre que rescata esta manera particular y propia de la tradición cultural constructiva de Caleta Tortel.

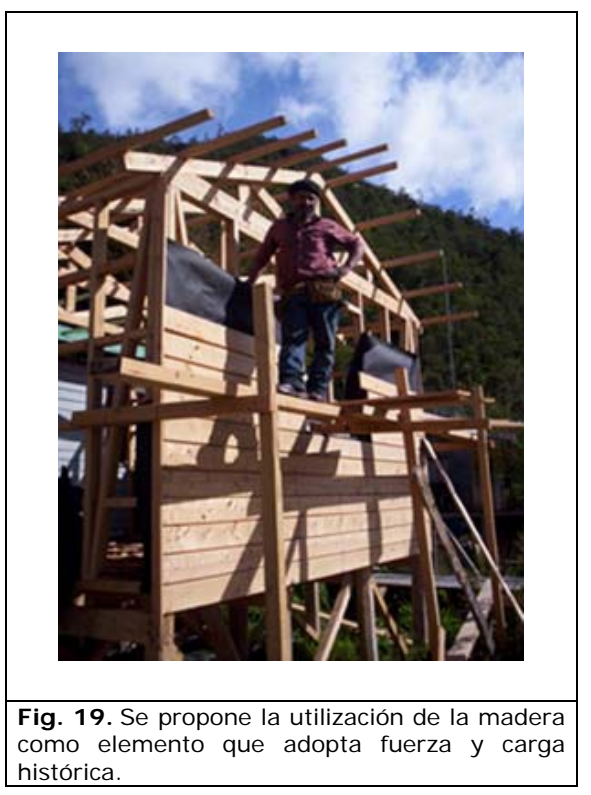




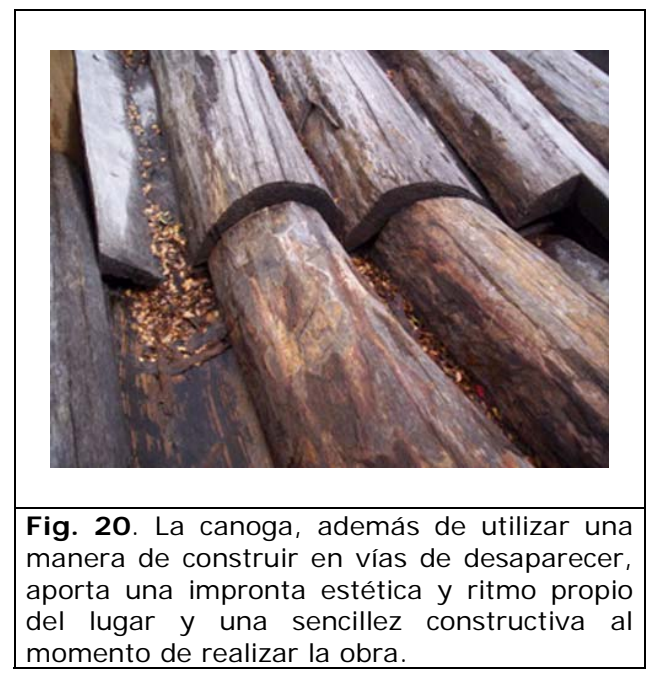

\subsection{Relación entorno construido}

\section{La arquitectura dialoga con el contexto temporal del territorio.}

El contexto. Un lugar específico y un tiempo dado.

-LOS AVANCES Y EL CRECIMIENTO DE LA LOCALIDAD: el proyecto reconoce el aumento de la población y acoge la necesidad de equipamientos y servicios para la población presente y próxima futura.

\section{Definición del proyecto}
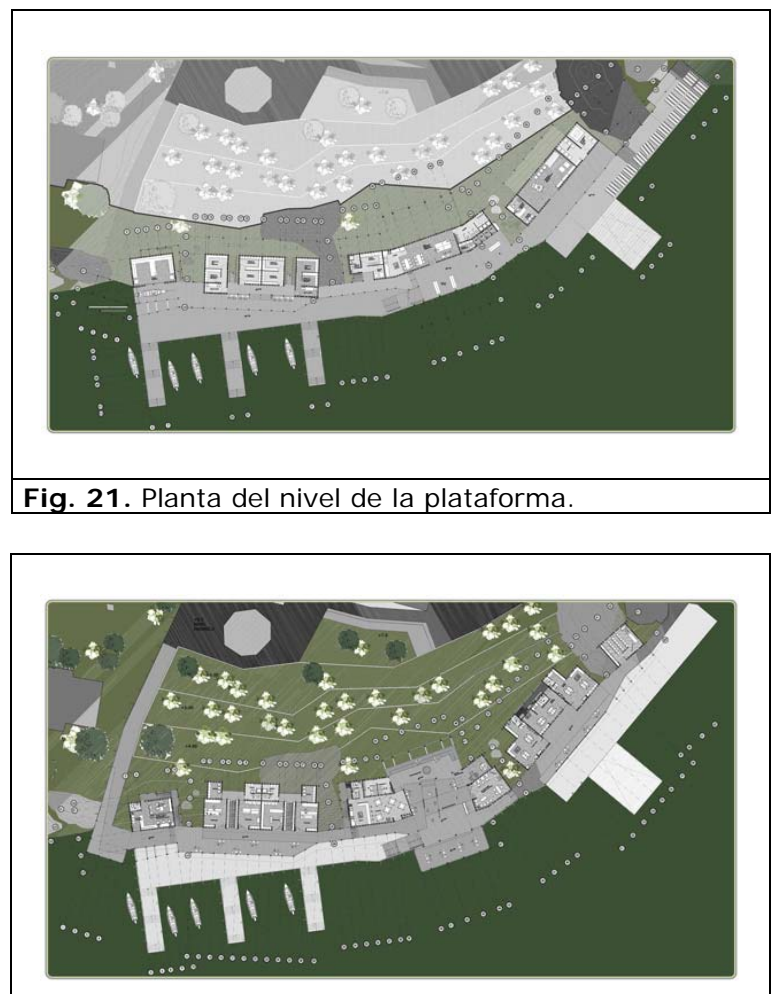

Fig. 22. Planta del nivel de la pasarela. 
El proyecto se conforma por una placa de conexión con el mar, donde se encuentra el embarcadero y la rampa, y por la continuidad de la pasarela a la cual se adosa el programa que activa este espacio público: mercado, talleres de confección artesanal, cocinerías, sala de difusión turística y cultural, servicios higiénicos, y los propios eventos generados por el espacio público propiamente tal. Es un lugar de encuentro y de flujos.

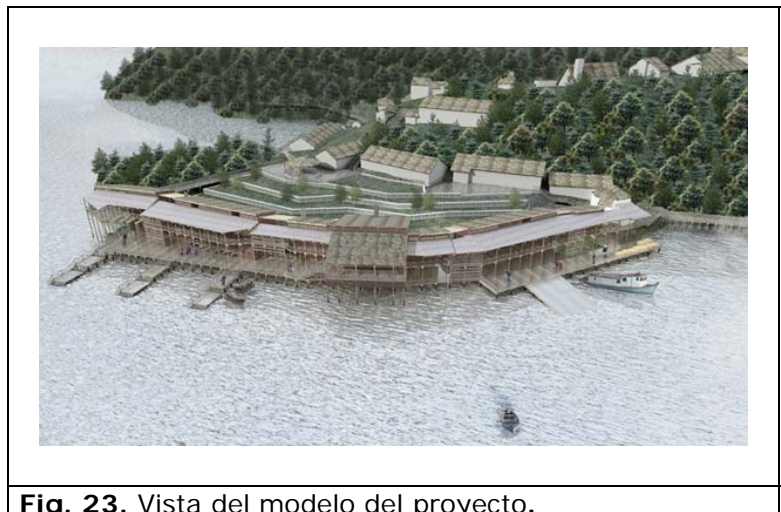

A la vez, el proyecto completa un circuito de equipamientos que conforman el sector centro de abastecimiento y servicios de la localidad, constituyendo el acceso marítimo a la comuna.

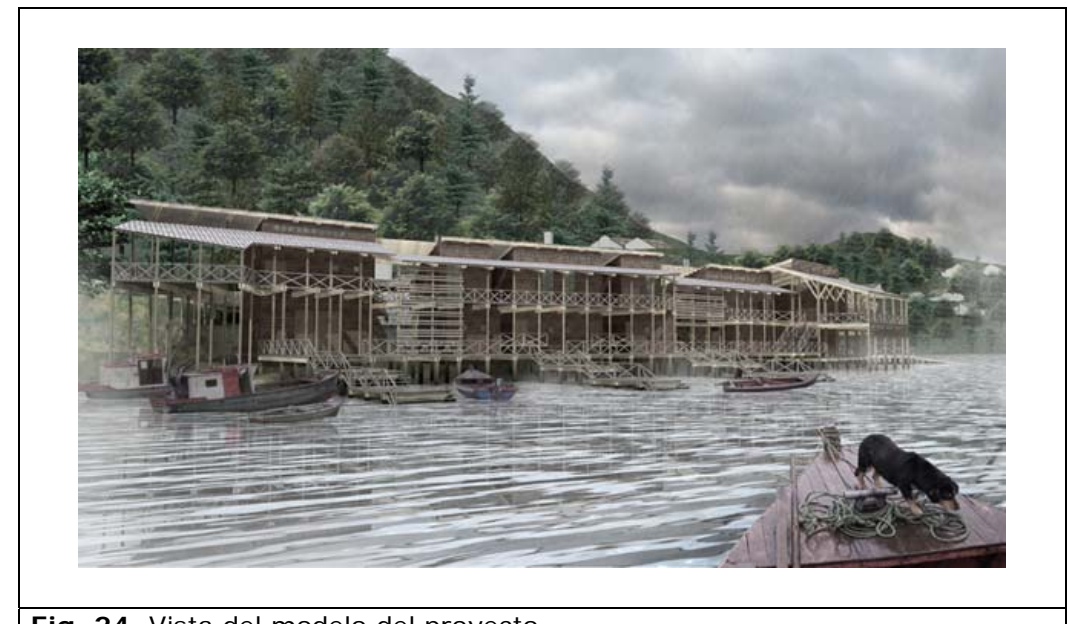

Fig. 24. Vista del modelo del proyecto.

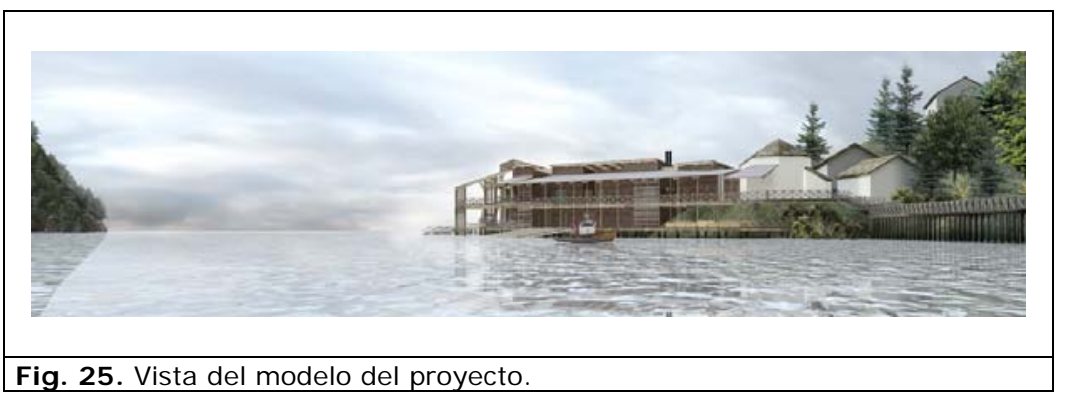



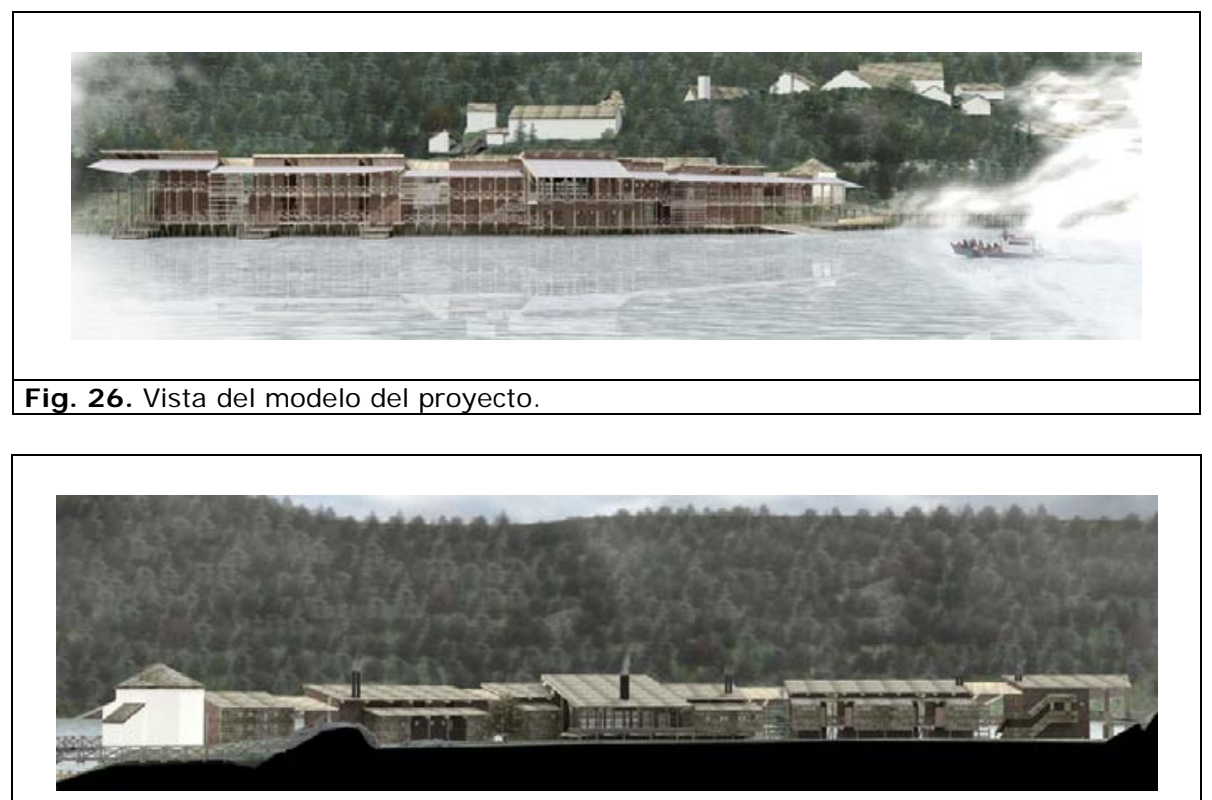

Fig. 27. Vista del modelo del proyecto.

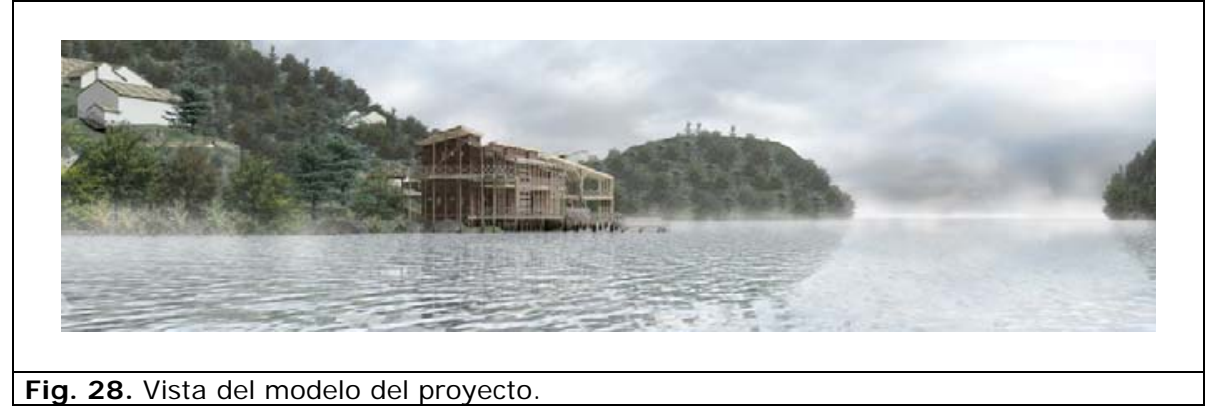

\section{Palabras finales}

El ejercicio de comprensión de una realidad distante y aislada, y de síntesis arquitectónica-urbana en este caso, dan cuenta de una valiosa experiencia, tanto en lo personal como en los profesional. El esfuerzo realizado con la guía del Profesor Arqto. J. Camplá, señala una vía posible para el abordaje de la arquitectura y urbanismo en un lugar de difícil asentamiento, en la perspectiva de la sustentabilidad. No obstante, el camino por recorrer es largo para efectos de llevar a la población de Caleta Tortel y su realidad a una nueva y mejor fase de desarrollo. Ella no puede concretarse sin la participación conjunta del Estado y del sector privado, tampoco sin la colaboración de las universidades del país cuya labor de investigación es imprescindible para poder efectivamente encaminarse hacia la sustentabilidad de las acciones en terreno. En especial, deberá abordarse con toda urgencia el estudio de los efectos del cambio climático en las regiones con ecosistemas de alta fragilidad y que, sin embargo, representan grandes reservas patrimoniales en el más amplio sentido de la palabra, para el futuro de la humanidad. 


\section{Bibliografía}

\section{Libros, $y$ artículos en revistas:}

-ALEXANDER, Christopher. El modo intemporal de construir. Ed.Gustavo Gili, Barcelona, 1981, 413 pág. Versión castellana de Iris Menéndez.

- AGUAYO, Carolina, Arquitecto Universidad de Chile y POT, Natacha, Arquitecto Universidad de Santiago de Chile. CHILE, MINISTERIO DE OBRAS PÚBLICAS, Dirección de Arquitectura, XI Región de Aysén. Bases técnicas de diseño para el instructivo de intervención de la Zona típica Caleta Tortel. Instructivo de Intervención, 2002.

-ELIASH, Humberto. "Arquitectura con-texto propio o ¿de qué contextualismo me habla?. En Revista DANA №26, Ed. Instituto Argentino de Investigaciones de Historia de la Arquitectura y el Urbanismo, ISSN 0326 - 8640, Buenos Aires, Argentina.

- GRES, Renato. "Características de los suelos del litoral de Aysén". Sociedad chilena de la Ciencia y el Suelo. (En prensa), 2005.

- HARgREAVES, María Paz. Caleta Tortel, Tradición de Habitar los Archipiélagos Australes. Ediciones Alto Volta. Santiago de Chile, 2005, 114 pág.

- HARTMANN S., Peter. "De Tortel a La Moneda, un largo camino". En REVISTA DE URBANISMO N4, digital, ISSN 0717-5051, D.Urbanismo F.A.U. Universidad de Chile, julio 2001, en índice DOAJ. [En línea] http://revistaurbanismo.uchile.cl

-HECHT, Romy. Trazado y territorio: Un estudio de los patrones fundacionales en Magallanes, Chile. Tesis de Magíster en Arquitectura Pontificia Universidad Católica de Chile, 1997.

-LOW, Setha. "Transformaciones del espacio público en la ciudad latinoamericana: cambios espaciales y prácticas sociales". En BIFURCACIONES, 14 pág. vol. 2, 2005. http://www.bifurcaciones.cl

- MARTNER, Carlos. "El Puerto y la vinculación entre lo local y lo global". En revista EURE V.25 N75, 1999.

- MORALES, José. Arquitectónica. Sobre la idea y el sentido de la arquitectura [1966-1969]. Biblioteca Nueva, Madrid, 1999, 222 pág.

- MUÑOZ, M. y SEGUEL, L. "Significado y trascendencia de lo efímero en lo cotidiano: Metamorfosis y abandono de la Plaza de Armas de Concepción". En PUBLICACIONES ARQCHILE.CL, ISSN 0718-431X, Departamento de Diseño y Teoría de la Arquitectura. Facultad de Arquitectura, Construcción y Diseño. Universidad del Bío Bío, 2001. http: ://www. Arqchile.cl.

- NACIONES UNIDAS. Declaración de Río. Conferencia de las Naciones Unidas sobre el Medio Ambiente y el Desarrollo, Río de Janeiro, Brasil, junio de 1992.

- PARCERISA, J osep y RUBERT, María. "La ciudad no es una hoja en blanco: hechos del urbanismo". En Ediciones ARQ, 2001. Santiago de Chile.

Seminarios de investigación tutelada, prácticas profesionales y memorias de título de Arquitecto:

- BARTOLOMÉ BRAVO, Pedro. Centro multiprogramático de desarrollo local Lago General Carrera, Chile. Memoria de Título, Facultad de Arquitectura y Urbanismo de la Universidad de Chile. Profesor Guía: Arqto. Andrés Elton, 2005.

- CAMPOSANO LUZZI, Macarena. Caleta y mercado pesqueros en Caldera. Memoria de Título, Facultad de Arquitectura y Urbanismo de la Universidad de Chile. Profesor Guía: Arqto. Leopoldo Prat, 2002.

-GONZÁLEZ, Rodrigo. Complejo pesquero y centro de capacitación Caleta la Vega: Calbuco, X Región. Memoria de Título, Facultad de Arquitectura y Urbanismo de la Universidad de Chile. Profesor Guía: Arqto. Humberto Eliash, 1997.

- HARTMANN S., Peter. "Práctica profesional en Urbanismo en Aisén, con una orientación verdaderamente universitaria". En: PAVEZ REYES, María Isabel. En la ruta de Juan Parrochia Beguin Premio Nacional de Urbanismo Chile 1996. Facultad de Arquitectura y Urbanismo - Vicerrectoría de Investigación y Desarrollo Universidad de Chile, Santiago, ISBN N956-19-0407-1, julio 2003, 319 págs., 465 ilustraciones, pp. 87-91.

- HARTMANN S., Peter. Antecedentes para un plan de desarrollo de Puerto Yungay. Memoria de práctica profesional, carrera de Arquitecto, Facultad de Arquitectura y Urbanismo de la Universidad de Chile. Prof. Guía Arqto. Juan Parrochia Beguin, 1982, 2 vol.

- HOPFNER KROMM, Heike. Estrategia de reactivación local, mediante la implementación de unidades microproductivas en Puerto I báñez, XI Región. Memoria de Título, Facultad de Arquitectura y Urbanismo de la Universidad de Chile. Profesor Guía: Arqto. Jorge Lobos, 2004.

- MEDEL SANTIBÁÑEZ, Paulina. Plaza Embarcadero Caleta Tortel, Tortel, Región de Aysén, Patagonia de Chile. Memoria de Proyecto de Título, Facultad de Arquitectura y Urbanismo, Universidad de Chile, Profesor Guía: Arqto. José Camplá Lehmann, 2007, 120 págs. [Extracto en el presente artículo].

- MEDEL SANTIBÁÑEZ, Paulina. Práctica Profesional en Caleta Tortel, XI Región de Aysén Patagonia de Chile. Memoria de práctica profesional, Facultad de Arquitectura y Urbanismo de la Universidad de Chile. Profesor Guía: Arqto. Luis Glodsack J., 2005.

- MERCADO FIGUEROA, Roberto. Plaza embarcadero Padre Antonio Ronchi, Caleta Tortel, Aysén, XI Región. Memoria de Título, Facultad de Arquitectura y Urbanismo de la Universidad de Chile. Profesor Guía: Arqto. Germán del Sol, 2003.

- MIRANDA MONSALVE, Fernando. Lugar de encuentro para pescadores artesanales: Puerto Aysén, XI Región. Memoria de Título, Facultad de Arquitectura y Urbanismo de la Universidad de Chile. Profesor Guía: Arqto. Mario Terán, 2000. 
-PULGAR PINAUD, Claudio. Habitat Kawésqar en Jetarkte, Puerto Edén, Archipiélago Patagónico Sur: viviendas, espacio público y equipamiento turístico cultural. Memoria de Título, Facultad de Arquitectura y Urbanismo de la Universidad de Chile. Profesor Guía: Arqto. Gustavo Munizaga V., 2006.

- QUIJADA MARTínEZ, Christián. Mercado fluvial de Puerto Aysén. Memoria de Título, Facultad de Arquitectura y Urbanismo de la Universidad de Chile. Profesor Guía: Arqto. Ernesto Calderón, 1998.

-SAN MARTÍN, Eduardo. "Regionalismo y modernidad apropiada en Chile". En ARQUITECTURAS DEL SUR $\mathrm{N}^{\circ} 14$, Revista de la Universidad del Bio-Bio, 2006.

-SUAREZ, Alejandro. Mercaleta: Recuperación del borde costero Coquimbo. Memoria de Título, Facultad de Arquitectura y Urbanismo de la Universidad de Chile. Profesor Guía: Arqto. José Camplá, 2002.

\section{Sitios Web:}

http://www.tortel.cl

http://caleta.tortel.free.fr

http://revistaurbanismo.uchile.cl/n4/hartmann/hartmann.html

http://elguanacovolador.blogspot.com

http:// www. municipalidaddetortel.cl/index.htm

http://www. moptt.cl

http://www.minvu.cl

http://www.patrimoniourbano.cl

http://www.corfo.cl/

http://www.rae.es

http://www.ecolyma.cl

http://www. patagoniainsular.cl/

http://arqecastillo. blogspot.com

http://www.meteochile.cl

http://www.chileportuario.cl

http://www.armada.cl

http://www.directemar.cl

http://www.solsan.cl

http://www. moptt.cl

http://www.indap.cl

http://www.dibam.cl

http://www.ecoceanos.cl

http://www.ecosur.org

http://www.rimisp.org

\section{Entrevistas:}

- Dra. Arqta. María Bertrand, Profesora de Urbanismo, F.A.U. Universidad de Chile. -Arqto. Luis Glodsack, Profesor de Ciencias de la Construcción, Universidad de Chile.- Iván Arellano, Capitán de Puerto de Caleta Tortel. -Rodrigo Maldonado, SECPLAN de la Municipalidad de Tortel.- Carlos Mansilla, Fomento Productivo de la Municipalidad de Tortel.

I mágenes, esquemas, diagramas ( $)$ :

Pedro Bartolomé (PB); María Paz Hargreaves (MH);Benjamín Strappa (BS); Paulina Medel (PM); .José Sánchez (JS). Todas las fotografías y dibujos son propiedad de las entidades o personas aquí nombradas, y no se permite su reproducción parcial o total sin autorización expresa. (c)

N. de R.: Paulina Andrea Medel Santibáñez, es arquitecta de la Universidad de Chile, titulada en la Facultad de Arquitectura y Urbanismo con el presente proyecto en 2007, con la Guía del Arqto. José Camplá Lehmann.

E-mail:<pmedel@improntaarquitectura.cl> 